\title{
LOCAL INTERPOLATION IN HILBERT SPACES OF DIRICHLET SERIES
}

\author{
JAN-FREDRIK OLSEN AND KRISTIAN SEIP
}

(Communicated by Joseph A. Ball)

\begin{abstract}
We denote by $\mathscr{H}$ the Hilbert space of ordinary Dirichlet series with square-summable coefficients. The main result is that a bounded sequence of points in the half-plane $\sigma>1 / 2$ is an interpolating sequence for $\mathscr{H}$ if and only if it is an interpolating sequence for the Hardy space $H^{2}$ of the same half-plane. Similar local results are obtained for Hilbert spaces of ordinary Dirichlet series that relate to Bergman and Dirichlet spaces of the half-plane $\sigma>1 / 2$.
\end{abstract}

\section{INTRODUCTION}

The aim of this paper is to shed more light on the function theory of the Hilbert space $\mathscr{H}$ that consists of all Dirichlet series of the form

$$
f(s)=\sum_{n=1}^{\infty} a_{n} n^{-s}
$$

( $s=\sigma+$ it a complex variable), with

$$
\|f\|_{\mathscr{H}}^{2}=\sum_{n=1}^{\infty}\left|a_{n}\right|^{2}<+\infty .
$$

We refer to [HLS97] and [HLS99] for the basics of $\mathscr{H}$. In particular, it should be stressed that $\mathscr{H}$ can be viewed as the Hardy space $H^{2}$ of the infinite-dimensional polydisk $\mathbb{D}^{\infty}$. From this perspective, when studying $\mathscr{H}$, we restrict to a onedimensional complex variety of $\mathbb{D}^{\infty}$. The intricacies of the function theory of $\mathscr{H}$ can be seen as reflecting this situation: We are dealing with a space of functions that "really" live on the infinite-dimensional set $\mathbb{D}^{\infty}$.

The definition of $\mathscr{H}$ shows that it consists of functions analytic in the half-plane $\sigma>1 / 2$. The key to understanding the local boundary behavior in this half-plane is the following embedding [Mon94, p. 140], [HLS97, Theorem 4.11]:

$$
\int_{\theta}^{\theta+1}\left|f\left(\frac{1}{2}+\mathrm{i} t\right)\right|^{2} \mathrm{~d} t \leq C\|f\|_{\mathscr{H}}^{2}
$$

Received by the editors July 17, 2006 and, in revised form, October 12, 2006.

2000 Mathematics Subject Classification. Primary 30B50; Secondary 30E05, 30H05, 42B30, $46 \mathrm{E} 20$.

The authors are supported by the Research Council of Norway grant 160192/V30. 
with $C$ an absolute constant independent of $\theta$. Of course, without further precautions, the embedding makes sense only when the Dirichlet series converges for $\sigma=1 / 2$. However, once (1.1) has been established for, say, Dirichlet polynomials, it follows that $f(s) / s$ is in $H^{2}$ of the half-plane $\sigma>1 / 2$ for every $f$ in $\mathscr{H}$. In other words, an implicit consequence of (1.1) is that $f$ has nontangential limits almost everywhere on the line $\sigma=1 / 2$, and we have therefore a well-defined boundary limit function which we by convention choose to denote by $f(1 / 2+\mathrm{i} t)$.

Another way of seeing a link between $\mathscr{H}$ and $H^{2}$ of the half-plane $\sigma>1 / 2$ is by comparing reproducing kernels. For $\mathscr{H}$, it is immediate that its kernel $k_{w}^{\mathscr{H}}(s)$ at the point $w$ is a translation of the Riemann zeta-function:

$$
k_{w}^{\mathscr{H}}(s)=\zeta(s+\bar{w}) .
$$

Since the zeta-function has a simple pole of residue 1 at $s=1$, we have

$$
k_{w}^{\mathscr{H}}(s)=\frac{1}{s+\bar{w}-1}+h(s+\bar{w})
$$

with $h$ an entire function. Here the first term is the kernel of $H^{2}$ of the half-plane $\sigma>1 / 2$ (properly normalized), and so (1.2) says that, near the diagonals, the kernels for the two spaces coincide modulo a bounded term.

In view of these observations, one might suspect that, locally, functions in $\mathscr{H}$ look like functions in $H^{2}$. The main result of this paper may be seen as a way of quantifying this similarity: Based on (1.1) and (1.2), we will prove that the interpolating sequences for the two spaces coincide, provided that we consider only bounded sequences of interpolation points.

The main result and its proof are presented in Section 2. We will then, in Section 3, briefly indicate that minor modifications of (1.1) and (1.2) yield similar interpolation results for a scale of Hilbert spaces studied by J. E. McCarthy in [McC04]. Section 4 contains some concluding remarks. In particular, we present there some simple observations on the problem of describing the unbounded interpolating sequences for $\mathscr{H}$, merely to hint at the complexity of the problem.

\section{LOCAL INTERPOLATION IN $\mathscr{H}$}

Let $H$ be a Hilbert space of functions on some set $\Omega$. We assume point evaluation $f \mapsto f(w)$ is bounded for each $w$ in $\Omega$ such that $H$ has a reproducing kernel. We denote this kernel by $k_{w}^{H}(s)$ and say that a sequence $S=\left(s_{j}\right)_{j=1}^{\infty}$ of distinct points $s_{j}$ from $\Omega$ is an interpolating sequence for $H$ if $f\left(s_{j}\right)=a_{j}$ has a solution $f$ in $H$ whenever $\left(a_{j} /\left\|k_{s_{j}}^{H}\right\|\right)_{j=1}^{\infty}$ is in $\ell^{2}$.

We set $\mathbb{C}_{1 / 2}=\{s=\sigma+\mathrm{i} t: \sigma>1 / 2\}$, and let $H^{2}\left(\mathbb{C}_{1 / 2}\right)$ denote the classical Hardy space of this half-plane. This is the space of all functions $f$ analytic in $\mathbb{C}_{1 / 2}$ with

$$
\|f\|_{H^{2}}^{2}=\sup _{\sigma>1 / 2} \frac{1}{2 \pi} \int_{-\infty}^{\infty}|f(\sigma+\mathrm{i} t)|^{2} \mathrm{~d} t<+\infty .
$$

We have nontangential boundary limits on the line $\sigma=1 / 2$ for almost every $t$ and may express the square of the norm as

$$
\|f\|_{H^{2}}^{2}=\frac{1}{2 \pi} \int_{-\infty}^{\infty}\left|f\left(\frac{1}{2}+\mathrm{i} t\right)\right|^{2} \mathrm{~d} t .
$$


The reproducing kernel of $H^{2}\left(\mathbb{C}_{1 / 2}\right)$ at $w$ is

$$
k_{w}^{H^{2}}(s)=\frac{1}{s+\bar{w}-1} .
$$

Our main result can now be stated as follows.

Theorem 2.1. Suppose $S$ is a bounded sequence of distinct points from $\mathbb{C}_{1 / 2}$. Then $S$ is an interpolating sequence for $\mathscr{H}$ if and only if it is an interpolating sequence for $H^{2}\left(\mathbb{C}_{1 / 2}\right)$.

Needless to say, now H. S. Shapiro and A. L. Shields's $H^{2}$ version [SS61] of L. Carleson's classical interpolation theorem [Car58] gives a geometric description of the bounded interpolating sequences for $\mathscr{H}$.

One implication is immediate from (1.2) and the fact that $f(s) / s$ is in $H^{2}\left(\mathbb{C}_{1 / 2}\right)$ whenever $f$ is in $\mathscr{H}$. Namely, when we solve the problem $f\left(s_{j}\right)=a_{j}$ with $f$ in $\mathscr{H}$, we simultaneously solve the problem $F(s)=a_{j} / s_{j}$ with $F$ in $H^{2}\left(\mathbb{C}_{1 / 2}\right)$. Also, since $S$ is bounded, $\left(a_{j} /\left\|k_{s_{j}}^{H^{2}}\right\|_{H^{2}}\right)_{j=1}^{\infty}$ is in $\ell^{2}$ if and only if $\left(s_{j} a_{j} /\left\|k_{s_{j}}^{\mathscr{H}}\right\|_{\mathscr{H}}\right)_{j=1}^{\infty}$ is in $\ell^{2}$.

Let us now assume that the bounded sequence $S$ is an interpolating sequence for $H^{2}\left(\mathbb{C}_{1 / 2}\right)$. We wish to prove that $S$ is also, then, an interpolating sequence for $\mathscr{H}$. To begin with, we observe that it suffices to show that the subsequence

$$
S_{\epsilon}=\left\{s_{j}=\sigma_{j}+\mathrm{i} t_{j} \in S: \frac{1}{2}<\sigma_{j} \leq \frac{1}{2}+\epsilon\right\}
$$

is an interpolating sequence for $\mathscr{H}$ for some small $\epsilon$. Indeed, it is clear that $S \backslash S_{\epsilon}$ is a finite sequence, which we may write as

$$
S \backslash S_{\epsilon}=\left(s_{j}\right)_{j=1}^{N} .
$$

The finite interpolation problem $f_{0}\left(s_{j}\right)=a_{j}, j=1, \ldots, N$ can be solved explicitly as follows. Choose primes $p_{1}, \ldots, p_{N}$ (not necessarily distinct) such that the product

$$
B(s)=\prod_{j=1}^{N}\left(1-p_{j}^{s_{j}-s}\right)
$$

has simple zeros at the points $s_{1}, \ldots, s_{N}$. If we set $B_{j}(s)=B(s) /\left(1-p_{j}^{s_{j}-s}\right)$, then the finite interpolation problem has solution

$$
f_{0}(s)=\sum_{j=1}^{N} a_{j} \frac{B_{j}(s)}{B_{j}\left(s_{j}\right)} .
$$

To solve the full interpolation problem $f\left(s_{j}\right)=a_{j}$, we can now solve

$$
f_{\epsilon}\left(s_{j}\right)=\frac{a_{j}-f_{0}\left(s_{j}\right)}{B\left(s_{j}\right)}, \quad s_{j} \in S_{\epsilon},
$$

so that we obtain the final solution $f=B f_{\epsilon}+f_{0}$. Clearly,

$$
\left(\frac{f_{\epsilon}\left(s_{j}\right)}{\left\|k_{s_{j}}^{\mathscr{H}}\right\|_{\mathscr{H}}}\right)_{s_{j} \in S_{\epsilon}} \in \ell^{2} \Longleftrightarrow\left(\frac{a_{j}}{\left\|k_{s_{j}}^{\mathscr{H}}\right\|_{\mathscr{H}}}\right)_{s_{j} \in S_{\epsilon}} \in \ell^{2},
$$

so that we have reduced the problem to showing that $S_{\epsilon}$ is an interpolating sequence for $\mathscr{H}$. 
Our reason for making the transition from $S$ to $S_{\epsilon}$ is that it will allow us to make use of the fact that

$$
\lim _{\epsilon \rightarrow 0} \sum_{s_{j} \in S_{\epsilon}}\left(\sigma_{j}-\frac{1}{2}\right)=0 .
$$

We note that (2.1) is just a consequence of the trivial fact that an interpolating sequence for $H^{2}\left(\mathbb{C}_{1 / 2}\right)$ is a Blaschke sequence in $\mathbb{C}_{1 / 2}$. Since $S$ is a bounded sequence, this means that

$$
\sum_{s_{j} \in S}\left(\sigma_{j}-\frac{1}{2}\right)<+\infty
$$

It seems difficult to obtain a direct solution of the interpolation problem since we do not have a reasonable substitute for Blaschke products. We will instead argue by duality, using the following lemma of R. P. Boas [You01], [BJ41].

Lemma 2.2. Suppose $\left(f_{j}\right)_{j=1}^{\infty}$ is a sequence of unit vectors in a Hilbert space $H$. Then the moment problem $\left\langle f, f_{j}\right\rangle_{H}=a_{j}$ has a solution $f$ in $H$ for every sequence $\left(a_{j}\right)_{j=1}^{\infty}$ in $\ell^{2}$ if and only if there is a positive constant $m$ such that

$$
\left\|\sum_{j} c_{j} f_{j}\right\|_{H} \geq m\left\|\left(c_{j}\right)\right\|_{\ell^{2}}
$$

for every finite sequence of scalars $\left(c_{j}\right)$.

Thus we need to prove (2.2) with $H=\mathscr{H}$ and $f_{j}=k_{s_{j}}^{\mathscr{H}} /\left\|k_{s_{j}}^{\mathscr{H}}\right\|_{\mathscr{H}}$ for $s_{j}$ in $S_{\epsilon}$.

To simplify the writing, we set $k_{s}=k_{s}^{\mathscr{H}}$ and suppress the index in the norm setting $\|f\|=\|f\|_{\mathscr{H}}$. Let $T$ be a positive number such that $\left|t_{j}\right| \leq T-1$ for every $s_{j}=\sigma_{j}+\mathrm{i} t_{j}$ in $S$. We start by using (1.1):

$$
\left\|\sum_{s_{j} \in S_{\epsilon}} c_{j} \frac{k_{s_{j}}}{\left\|k_{s_{j}}\right\|}\right\|^{2} \geq m_{T} \int_{-T}^{T}\left|\sum_{s_{j} \in S_{\epsilon}} c_{j} \frac{k_{s_{j}}\left(\mathrm{i} t+\frac{1}{2}\right)}{\left\|k_{s_{j}}\right\|}\right|^{2} \mathrm{~d} t .
$$

The trick is now to replace the kernels of $\mathscr{H}$ by the kernels of $H^{2}\left(\mathbb{C}_{1 / 2}\right)$. We use (1.2) and the triangle inequality:

$$
\begin{gathered}
\left(\int_{-T}^{T}\left|\sum_{s_{j} \in S_{\epsilon}} c_{j} \frac{k_{s_{j}}\left(\mathrm{i} t+\frac{1}{2}\right)}{\left\|k_{s_{j}}\right\|}\right|^{2} \mathrm{~d} t\right)^{1 / 2} \geq\left(\int_{-T}^{T}\left|\sum_{s_{j} \in S_{\epsilon}} c_{j} \frac{\left\|k_{s_{j}}\right\|^{-1}}{\mathrm{i} t+\bar{s}_{j}-\frac{1}{2}}\right|^{2} \mathrm{~d} t\right)^{1 / 2} \\
-\left(\int_{-T}^{T}\left|\sum_{s_{j} \in S_{\epsilon}} c_{j} h\left(\mathrm{i} t+\bar{s}_{j}+\frac{1}{2}\right)\left\|k_{s_{j}}\right\|^{-1}\right|^{2} \mathrm{~d} t\right)^{1 / 2} .
\end{gathered}
$$

We split the first term on the right into two pieces:

$$
\int_{-T}^{T}\left|\sum_{s_{j} \in S_{\epsilon}} c_{j} \frac{\left\|k_{s_{j}}\right\|^{-1}}{\mathrm{i} t+\bar{s}_{j}-\frac{1}{2}}\right|^{2} \mathrm{~d} t=\left(\int_{-\infty}^{\infty}-\int_{|t|>T}\right)\left|\sum_{s_{j} \in S_{\epsilon}} c_{j} \frac{\left\|k_{s_{j}}\right\|^{-1}}{\mathrm{i} t+\bar{s}_{j}-\frac{1}{2}}\right|^{2} \mathrm{~d} t
$$


The point is now that the first term on the right in (2.4) is just

$$
2 \pi\left\|\sum_{s_{j} \in S_{\epsilon}} c_{j} \frac{k_{s_{j}}}{\left\|k_{s_{j}}\right\|}\right\|_{H^{2}}^{2}
$$

so that by using the hypothesis on $S$ and Lemma 2.2 , we arrive at the inequality

$$
\begin{gathered}
\frac{1}{m_{T}}\left\|\sum_{s_{j} \in S_{\epsilon}} c_{j} \frac{k_{s_{j}}}{\left\|k_{s_{j}}\right\|}\right\| \geq m^{\prime}\left\|\left(c_{j}\right)\right\|_{\ell^{2}}-\left(\int_{-T}^{T}\left|\sum_{s_{j} \in S_{\epsilon}} c_{j} h\left(\mathrm{i} t+\bar{s}_{j}+\frac{1}{2}\right)\left\|k_{s_{j}}\right\|^{-1}\right|^{2} \mathrm{~d} t\right)^{1 / 2} \\
-\left(\int_{|t|>T}\left|\sum_{s_{j} \in S_{\epsilon}} c_{j} \frac{\left\|k_{s_{j}}\right\|^{-1}}{\mathrm{i} t+\bar{s}_{j}-\frac{1}{2}}\right|^{2} \mathrm{~d} t\right)^{1 / 2} .
\end{gathered}
$$

The two terms that are subtracted on the right are easily estimated. Indeed, by applying the triangle inequality to get the sums outside of the integrals and then the Cauchy-Schwarz inequality, we see that the first term is bounded by

$$
\sum_{s_{j} \in S_{\epsilon}}\left|c_{j}\right|\left\|k_{s_{j}}\right\|^{-1}\left(\int_{-T}^{T}\left|h\left(\mathrm{i} t+\bar{s}_{j}-\frac{1}{2}\right)\right|^{2} \mathrm{~d} t\right)^{1 / 2} \leq B\left(\sum_{s_{j} \in S_{\epsilon}}\left\|k_{s_{j}}\right\|^{-2}\right)^{1 / 2}\left\|\left(c_{j}\right)\right\|_{\ell^{2}}
$$

with $B$ depending only on $h, S$, and $T$. The second term is treated in a similar way, and we find that it is bounded by

$$
\sum_{s_{j} \in S_{\epsilon}}\left|c_{j}\right|\left\|k_{s_{j}}\right\|^{-1}\left(\int_{|t|>T}\left|\frac{1}{\bar{s}_{j}-\frac{1}{2}+\mathrm{i} t}\right|^{2} \mathrm{~d} t\right)^{1 / 2} \leq 2\left(\sum_{s_{j} \in S_{\epsilon}}\left\|k_{s_{j}}\right\|^{-2}\right)^{1 / 2}\left\|\left(c_{j}\right)\right\|_{\ell^{2}} .
$$

By (1.2), $\left\|k_{s_{j}}\right\|^{-2} \leq C\left(\sigma_{j}-1 / 2\right)$ with $C$ depending only on $S$. In view of $(2.1)$, we obtain (2.2) by choosing $\epsilon$ sufficiently small. This completes the proof of the theorem.

\section{Local interpolation in MCCARThy's SPACES $\mathscr{H}_{\alpha}$}

In [McC04], McCarthy studied a wider class of Hilbert spaces of Dirichlet series. In particular, he found a scale of such spaces that resembles the familiar scale to which the classical Dirichlet, Hardy, and Bergman spaces belong. We shall now see that, as far as local interpolation is concerned and in accordance with what was done above, these spaces can be linked to their classical counterparts in the half-plane $\sigma>1 / 2$.

We declare $\mathscr{H}_{\alpha}$ with $\alpha \leq 1$ to be the space of all Dirichlet series of the form

$$
f(s)=\sum_{n=1}^{\infty} a_{n} n^{-s}
$$

such that

$$
\|f\|_{\mathscr{H}_{\alpha}}^{2}=\sum_{n=1}^{\infty}\left|a_{n}\right|^{2} \log ^{\alpha}(n+1)<+\infty
$$

We observe that every space $\mathscr{H}_{\alpha}$ consists of functions analytic in the half-plane $\sigma>1 / 2$. For each $\alpha$, the basic estimate (1.1) can now be transformed into a version that reveals the local boundary behavior of functions in $\mathscr{H}_{\alpha}$. To this end, 
let $\mathrm{d} m$ denote Lebesgue area measure and $Q_{\theta}$ the half-strip $\sigma>1 / 2, \theta<t<\theta+1$. Then, writing (1.1) as

$$
\int_{\theta}^{\theta+1}|f(\sigma+\mathrm{i} t)|^{2} \mathrm{~d} t \leq C \sum_{n=1}^{\infty}\left|a_{n}\right|^{2} n^{2 \sigma-1},
$$

multiplying both sides by $\left(\sigma-\frac{1}{2}\right)^{-\alpha-1}$, and integrating from $\frac{1}{2}$ to $+\infty$, we get for $\alpha<0$ :

$$
\int_{Q_{\theta}}|f(s)|^{2}\left(\sigma-\frac{1}{2}\right)^{-\alpha-1} \mathrm{~d} m(s) \leq C\|f\|_{\mathscr{H}_{\alpha}}^{2} .
$$

By a similar computation for $0<\alpha \leq 1$, we obtain

$$
\int_{Q_{\theta}}\left|f^{\prime}(s)\right|^{2}\left(\sigma-\frac{1}{2}\right)^{-\alpha+1} \mathrm{~d} m(s) \leq C\|f\|_{\mathscr{H}_{\alpha}}^{2} .
$$

The natural counterparts to the spaces $\mathscr{H}_{\alpha}$ in the half-plane $\sigma>1 / 2$ are therefore the corresponding Dirichlet-type spaces $(0<\alpha \leq 1)$ and Bergman spaces $(\alpha<0)$. As there is essentially nothing new in this discussion, we will be brief with the technical details, and some of them will be left to the reader.

Along with $\mathscr{H}_{\alpha}$, we define the space $D_{\alpha}\left(\mathbb{C}_{1 / 2}\right)$ as follows. For $\alpha<0$, it is the weighted Bergman space of functions analytic in $\mathbb{C}_{1 / 2}$ such that

$$
\|f\|_{D_{\alpha}}^{2}=\frac{1}{\pi} \int_{\mathbb{C}_{1 / 2}}|f(s)|^{2}\left(\sigma-\frac{1}{2}\right)^{-\alpha-1} \mathrm{~d} m(s)<+\infty .
$$

For $0<\alpha<1$, we let $D_{\alpha}\left(\mathbb{C}_{1 / 2}\right)$ be the Dirichlet-type space of functions analytic in $\mathbb{C}_{1 / 2}$ such that $f(\sigma) \rightarrow 0$ when $\sigma \rightarrow \infty$ and

$$
\|f\|_{D_{\alpha}}^{2}=\frac{1}{\pi} \int_{\mathbb{C}_{1 / 2}}\left|f^{\prime}(s)\right|^{2}\left(\sigma-\frac{1}{2}\right)^{-\alpha+1} \mathrm{~d} m(s)<+\infty .
$$

Finally, we declare $D_{1}\left(\mathbb{C}_{1 / 2}\right)$ to be the Dirichlet space in $\mathbb{C}_{1 / 2}$, or more precisely, the collection of functions $f$ analytic in $\mathbb{C}_{1 / 2}$ such that

$$
\|f\|_{D_{1}}^{2}=\frac{1}{2 \pi} \int_{-\infty}^{\infty}\left|f\left(\frac{1}{2}+\mathrm{i} t\right)\right|^{2} \frac{\mathrm{d} t}{1+t^{2}}+\frac{1}{\pi} \int_{\mathbb{C}_{1 / 2}}\left|f^{\prime}(s)\right|^{2} \mathrm{~d} m(s)<+\infty .
$$

The reproducing kernel for $D_{\alpha}\left(\mathbb{C}_{1 / 2}\right)$ at $w$ is

$$
k_{w}^{D_{\alpha}}(s)=c_{\alpha}(\bar{w}+s-1)^{\alpha-1}
$$

when $\alpha<1$, with $c_{\alpha}=(-\alpha) 2^{-\alpha-1}$ for $\alpha<0$ and $c_{\alpha}=2^{\alpha-1}(1-\alpha)^{-1}$ for $0<\alpha<1$. In the limiting case $\alpha=1$, we have

$$
k_{w}^{D_{1}}(s)=\frac{3-2 \bar{w}}{1-2 \bar{w}} \frac{3+2 s}{1+2 s}\left(\log \frac{(1+2 \bar{w})(1+2 s)}{2^{3}}+\log \frac{1}{\bar{w}+s-1}\right) .
$$

What is essential here is that for $s$ and $w$ in a bounded set, we have

$$
k_{w}^{D_{1}}(s)=\log \frac{1}{s+\bar{w}-1}+\text { a bounded term. }
$$

Returning to $\mathscr{H}_{\alpha}$, we observe that its reproducing kernel may be expressed in terms of a weighted zeta-function,

$$
k_{w}^{\mathscr{H}_{\alpha}}(s)=\sum_{n=1}^{\infty} \frac{n^{-s-\bar{w}}}{\log ^{\alpha}(n+1)} .
$$


As for the classical zeta-function, we have a singularity at $s=1$, in agreement with the behavior of the kernel of $D_{\alpha}\left(\mathbb{C}_{1 / 2}\right)$.

Lemma 3.1. For $\alpha<1$, we have

$$
\sum_{n=1}^{\infty} \frac{n^{-s}}{\log ^{\alpha}(n+1)}=\Gamma(1-\alpha)(s-1)^{\alpha-1}+\mathcal{O}(1)
$$

as $s \rightarrow 1$. We also have

$$
\sum_{n=1}^{\infty} \frac{n^{-s}}{\log (n+1)}=\log \frac{1}{s-1}+\mathcal{O}(1)
$$

as $s \rightarrow 1$.

Proof. The proof is a calculation analogous to the one for the Riemann zeta-function found for instance in [Ivi03]. To begin with,

$$
\begin{aligned}
\sum_{n=1}^{\infty} \frac{n^{-s}}{\log ^{\alpha}(n+1)} & =\int_{1}^{\infty} \frac{x^{-s}}{\log ^{\alpha}(x+1)} \mathrm{d}[x] \\
& =\int_{1}^{\infty} \frac{x^{-s-1}[x]}{\log ^{\alpha}(x+1)}\left(s+\frac{\alpha}{\log (x+1)} \frac{x}{x+1}\right) \mathrm{d} x
\end{aligned}
$$

The integral

$$
\begin{aligned}
\int_{1}^{\infty}\left[\frac{x^{-s-1}[x]}{\log ^{\alpha}(x+1)}\left(s+\frac{\alpha}{\log (x+1)} \frac{x}{x+1}\right)\right. & \\
& \left.-\frac{x^{-s}}{\log ^{\alpha}(x+1)}\left(s+\frac{\alpha}{\log (x+1)}\right)\right] \mathrm{d} x
\end{aligned}
$$

converges absolutely and defines an analytic function in the right half-plane. We may therefore pass from $[x]$ to $x$ in our integral, and ignore the factor $x /(x+1)$. For a similar reason, we may replace $\log (x+1)$ by $\log x$ and, if necessary, change the lower limit of integration.

When $\alpha<1$, we make the following computation:

$$
\int_{1}^{\infty} \frac{x^{-s}}{\log ^{\alpha} x} \mathrm{~d} x=\Gamma(1-\alpha)(s-1)^{\alpha-1} .
$$

This gives the desired result for $0<\alpha<1$. When $\alpha<0$, we find, using the functional equation for the gamma-function, that

$$
\int_{1}^{\infty} \frac{x^{-s}}{\log ^{\alpha} x}\left(s+\frac{\alpha}{\log x}\right) \mathrm{d} x=\Gamma(1-\alpha)(s-1)^{\alpha-1}
$$

as well. In the limiting case $\alpha=1$, we find that

$$
\int_{2}^{\infty} \frac{x^{-s}}{\log x} \mathrm{~d} x=\log \frac{1}{s-1}+\mathcal{O}(1)
$$

as $s \rightarrow 1$.

We have now found the appropriate analogues of the basic relations (1.1) and (1.2). The proof of the following extension of Theorem 2.1 is essentially a plain rewriting of the proof in Section 2. We trust that the interested reader will check the details. 
Theorem 3.2. Suppose $S$ is a bounded sequence of distinct points from $\mathbb{C}_{1 / 2}$ and assume $\alpha \leq 1$. Then $S$ is an interpolating sequence for $\mathscr{H}_{\alpha}$ if and only if it is an interpolating sequence for $D_{\alpha}\left(\mathbb{C}_{1 / 2}\right)$.

A small technical remark is in order. One may distinguish between interpolating sequences as defined above and so-called universal interpolating sequences, i.e., sequences $\left(s_{j}\right)_{j=1}^{\infty}$ for which $f \mapsto\left(f\left(s_{j}\right) /\left\|k_{s_{j}}^{H}\right\|_{H}\right)$ maps $H$ both into and onto $\ell^{2}$. In the latter case, one then has the Carleson embedding

$$
\sum_{j=1}^{\infty}\left|f\left(s_{j}\right)\right|^{2}\left\|k_{s_{j}}^{H}\right\|_{H}^{-2} \leq C\|f\|_{H}^{2}
$$

with some positive constant $C$. In the case of bounded interpolating sequences for $\mathscr{H}$, there is no reason to make a distinction because every bounded interpolating sequence for $\mathscr{H}$ is also a universal interpolating sequence for $\mathscr{H}$. The same holds true for $\mathscr{H}_{\alpha}$ when $\alpha<0$. However, for $\mathscr{H}_{\alpha}$ with $0<\alpha \leq 1$, this is no longer the case [Bis94], [MS93], and one should therefore make a distinction. Still, it is plain that Theorem 3.2 remains valid if one replaces each occurrence of the string "an interpolating sequence" by "a universal interpolating sequence".

There exist geometric descriptions of the (universal) interpolating sequences for all $\alpha \leq 1$. For $\alpha<0$, Beurling-type density theorems were proved in [Sei93]. Descriptions in terms of Carleson measures were found by W. Cohn in the case $0<\alpha<1$ [Coh93] and independently by C. Bishop and by D. Marshall and C. Sundberg in the case $\alpha=1$ [Bis94], [MS93]. For further information, we refer to the monograph [Sei04].

\section{Concluding Remarks}

It may be noted that Theorem 2.1 gives the first general sufficient condition for zero sequences of functions in $\mathscr{H}$. The statement is that for each bounded interpolating sequence $S$ for $H^{2}\left(\mathbb{C}_{1 / 2}\right)$, there is a function $f$ in $\mathscr{H}$ vanishing on $S$. On the other hand, a simple argument ${ }^{1}$ shows that this sequence $S$ cannot be the zero sequence of any function in $\mathscr{H}$. Indeed, the almost periodicity of the function $t \mapsto f(\sigma+\mathrm{i} t)$ along with Rouché's theorem implies that for any zero $\sigma_{0}+\mathrm{i} t_{0}$ of a function $f$ in $\mathscr{H}$ and $0<\epsilon<\sigma$, there is a positive number $T$ such that every rectangle $\left|\sigma-\sigma_{0}\right|<\epsilon, \theta<t<\theta+T$ contains a zero of $f$. In particular, this means that no function in $\mathscr{H}$ has a nonempty finite zero sequence.

We finally make some remarks about unbounded interpolating sequences for $\mathscr{H}$. Take first an arbitrary interpolating sequence located on the real line. We may assume with no essential loss of generality that the sequence is $\sigma_{j}=1 / 2+2^{-j}$, $j=1,2,3, \ldots$. Then any sequence $s_{j}=\sigma_{j}+\mathrm{i} t_{j}$ will give us a Carleson embedding of the form (3.3). To see this, we associate with

$$
f(s)=\sum_{n=1}^{\infty} a_{n} n^{-s}
$$

the Dirichlet series

$$
f^{+}(s)=\sum_{n=1}^{\infty}\left|a_{n}\right| n^{-s} .
$$

\footnotetext{
${ }^{1}$ We thank E. Saksman for bringing this point to our attention.
} 
This means that $\left|f\left(s_{j}\right)\right| \leq f^{+}\left(\sigma_{j}\right)$, and since $\|f\|_{\mathscr{H}}=\left\|f^{+}\right\|_{\mathscr{H}}$, we obtain the result from the fact that $\left(\sigma_{j}\right)_{j=1}^{\infty}$ yields a Carleson embedding.

We next observe that the sequence $\left(s_{j}\right)_{j=1}^{\infty}$ can be split into a finite number of interpolating sequences for $\mathscr{H}$. (The number of sequences depends only on $\left(\sigma_{j}\right)_{j=1}^{\infty}$.) This follows from Gerschgorin's circle theorem. Indeed, Lemma 2.2 says that it is enough to check that the normalized Grammian

$$
\left(\frac{k_{s_{j}}^{\mathscr{H}}\left(s_{l}\right)}{\left\|k_{s_{j}}^{\mathscr{H}}\right\|_{\mathscr{H}}\left\|k_{s_{l}}^{\mathscr{H}}\right\|_{\mathscr{H}}}\right)_{j, l=1}^{\infty}
$$

is invertible as a map on $\ell^{2}$. We observe that in our case the entries of the matrix decay exponentially and monotonically away from the main diagonal. Thus by splitting $\left(\sigma_{j}\right)_{j=1}^{\infty}$ into sufficiently sparse subsequences, we obtain the invertibility from Gerschgorin's criterion.

To illustrate a different point, we construct the following sequence. For each positive integer $j$, pick points equi-distributed on the line segment $\sigma=1 / 2+2^{-j}$, $0 \leq t \leq 1$; i.e., choose

$$
s_{j, l}=\frac{1}{2}+2^{-j}+\mathrm{i} \frac{l}{j}, \quad l=1,2, \ldots, j .
$$

Then Carleson's theorem along with our Theorem 2.1 shows that $\left(s_{j, l}\right)$ is an interpolating sequence for $\mathscr{H}$. In particular, the Carleson embedding (3.3) holds. Now if we move the points vertically and far apart, the Carleson embedding may fail. This is a consequence of the almost periodicity of $t \mapsto \zeta(\sigma+\mathrm{i} t)$. If we measure the distance between two points in terms of the angle between the corresponding reproducing kernels, this almost periodicity implies that points that are far apart in the hyperbolic sense of the half-plane may be arbitrarily close in the geometry induced by $\mathscr{H}$.

The conclusion is that the nature of the problem changes quite dramatically when we remove the a priori assumption that the sequence $S$ be bounded. Any nontrivial results about unbounded interpolating sequences and Carleson measures for $\mathscr{H}$ should yield interesting information about the global behavior of functions in $\mathscr{H}$.

\section{REFERENCES}

[Bis94] C. Bishop, Interpolating sequences for the Dirichlet space and its multipliers, Preprint, 1994.

[BJ41] R. P. Boas Jr., A general moment problem, Amer. J. Math. 63, 361-370 (1941). MR0003848 (2:281d)

[Car58] L. Carleson, An interpolation problem for bounded analytic functions, Amer. J. Math. 80, 921-930 (1958). MR0117349 (22:8129)

[Coh93] W. Cohn, Interpolation and multipliers on Besov and Sobolev spaces, Complex Variables Theory Appl. 22, 35-45 (1993). MR1277009 (95g:30069)

[HLS97] H. Hedenmalm, P. Lindqvist and K. Seip, A Hilbert space of Dirichlet series and systems of dilated functions in $L^{2}(0,1)$, Duke Math. J. 86, 1-37 (1997). MR1427844 (99i:42033)

[HLS99] H. Hedenmalm, P. Lindqvist and K. Seip, Addendum to "A Hilbert space of Dirichlet series and systems of dilated functions in $L^{2}(0,1)$ ", Duke Math. J. 99, 175-178 (1999). MR1700745 (2000g:42029)

[Ivi03] A. Ivic, The Riemann Zeta-Function. Theory and Applications, Dover Publications Inc., 2003. MR1994094

[McC04] J. E. McCarthy, Hilbert spaces of Dirichlet series and their multipliers, Trans. Amer. Math. Soc. 356(3), 881-893 (2004). MR1984460 (2004j:30006) 
[Mon94] H. L. Montgomery, Ten Lectures on the Interface Between Analytic Number Theory and Harmonic Analysis, volume 84 of CBMS Regional Conference Series in Mathematics, AMS, 1994.

[MS93] D. E. Marshall and C. Sundberg, Interpolating sequences for the multipliers of the Dirichlet space, Preprint. Availiable at http://www.math.washington.edu/ marshall/ preprints/preprints.html, 1993.

[Sei93] K. Seip, Beurling type density theorems in the unit disk, Invent. Math. 113, 21-39 (1993). MR1223222 (94g:30033)

[Sei04] K. Seip, Interpolation and Sampling in Spaces of Analytic Functions, volume 33 of University Lecture Series, American Mathematical Society, Providence, R. I., 2004. MR2040080 (2005c:30038)

[SS61] H. S. Shapiro and A. L. Shields, On some interpolation problems for analytic functions, Amer. J. Math. 83, 513-532 (1961). MR0133446 (24:A3280)

[You01] R. M. Young, An Introduction to Nonharmonic Fourier Series, Academic Press, New York, Revised First Edition, 2001. MR1836633 (2002b:42001)

Department of Mathematics, Washington University in St. Louis, St. Louis, MisSOURI 63130

E-mail address: janfreol@math.ntnu.no

Department of Mathematical Sciences, Norwegian University of Science and TechNology (NTNU), NO-7491 Trondheim, Norway

E-mail address: seip@math.ntnu.no 